山्山FFRANÇAISE

$\gg \mathrm{DE}$

필 PEGOGIE

\section{Revue française de pédagogie}

Recherches en éducation

156 | juillet-septembre 2006

Les espaces locaux d'interdépendance entre

établissements : une comparaison européenne

\title{
TANGUY Lucie. Les instituts du travail : la formation syndicale à l'université de 1955 à nos jours
}

Rennes : Presses universitaires de Rennes, 2006. - 256 p. (Des sociétés)

\section{Alain Baubion-Broye}

\section{OpenEdition}

Édition électronique

URL : http://journals.openedition.org/rfp/689

DOI : $10.4000 /$ rfp.689

ISSN : 2105-2913

Éditeur

ENS Éditions

Édition imprimée

Date de publication : 1 juillet 2006

Pagination : 192-193

ISBN : 978-2-7342-1060-3

ISSN : 0556-7807

Référence électronique

Alain Baubion-Broye, "TANGuY Lucie. Les instituts du travail : la formation syndicale à l'université de 1955 à nos jours », Revue française de pédagogie [En ligne], 156 I juillet-septembre 2006, mis en ligne le 24 septembre 2010, consulté le 22 septembre 2020. URL : http://journals.openedition.org/rfp/689 ; DOI : https://doi.org/10.4000/rfp.689

Ce document a été généré automatiquement le 22 septembre 2020

(c) tous droits réservés 


\title{
TANGUY Lucie. Les instituts du travail : la formation syndicale à l'université de 1955 à nos jours
}

Rennes : Presses universitaires de Rennes, 2006. - 256 p. (Des sociétés)

\author{
Alain Baubion-Broye
}

\section{RÉFÉRENCE}

TANGUY Lucie. Les instituts du travail : la formation syndicale à l'université de 1955 à nos jours. Rennes : Presses universitaires de Rennes, 2006. - 256 p. (Des sociétés).

1 Cet ouvrage vient, pour sûr, instruire sur une réalité mal connue (ou méconnue), celle de la formation ouvrière à laquelle certaines universités françaises proposent des cadres d'insertion et de développement. À ce jour, existent deux instituts nationaux et neuf instituts régionaux (le dernier a été créé au sein de l'université de Toulouse-Le Mirail en 2003).

2 On trouvera dans cet ouvrage dû à la sociologue Lucie Tanguy une tentative rigoureuse et résolue pour retracer l'histoire de ces instituts du travail depuis 1955, date fondatrice de l'institut de Strasbourg intégré à la Faculté de Droit et des Sciences politiques, avec pour directeur Marcel David.

3 L'ouvrage étudie les conditions et les facteurs sociohistoriques et idéologiques qui ont favorisé l'émergence de ces institutions, véritables innovations dans le champ de la formation syndicale. Il montre le rôle pionnier d'acteurs, issus des mouvements chrétiens, socialistes, marxistes, de la résistance à l'oppression nazie, porteurs d'un projet humaniste, convaincus que l'idéal de la démocratie devait avoir pour corrélat une action culturelle unificatrice, respectueuse des diversités. Sans doute un tel idéal rejoint-il les exigences de réforme auxquelles en d'autres contextes, M. Monod, proche du ministre J. Zay en 1936, plus tard le psychologue $\mathrm{H}$. Wallon et le physicien P. Langevin ont fortement contribué. Pour tous ces acteurs, malgré les différences que 
Lucie Tanguy souligne et explique, la connaissance, en particulier celle qui a trait à la législation et au droit du travail (introduits dans l'enseignement universitaire dans les années 1950) est indispensable au syndicalisme. Elle est un garant de la justice sociale. Les «missionnaires de la cause syndicale " (selon les termes de l'auteur), les directeurs influents d'instituts du travail tels David, Bartoli, Sellier étaient, par leur formation intellectuelle et dans leur fonction universitaire, des spécialistes d'économie et de droit. Si l'on se reporte à une partie de l'arrêté qui porte création du premier institut à Strasbourg, on perçoit quelques uns des axes principaux auxquels l'ensemble des instituts actuels, peu ou prou, se réfèrent dans leurs statuts et leurs pratiques. L'accent y est mis sur le développement de recherches et d'enseignements relatifs aux sciences du travail, sur l'organisation de sessions de formation à l'adresse de cadres du monde du travail et de colloques sur des thèmes économiques, législatifs, techniques, sur la documentation (cf. p. 46). L'auteur consacre utilement un chapitre de son ouvrage à analyser ce qui, à la fois lie et spécifie les instituts dans le système universitaire et le monde du travail et du syndicalisme en France. Selon elle, de leur situation singulière résultent des risques de marginalité relative. Mais elle montre qu'en raison même de leur situation les instituts ont permis, avec des fortunes et des résultats divers, une solide expérimentation de nouveaux rapports enseignants-enseignés, de nouveaux types de coopération et d'ajustement entre acteurs universitaires et des confédérations syndicales représentatives impliquées ensemble dans l'histoire des instituts du travail. Sont examinés, de façon précise et informée, les déterminants et les différents aspects de la " pédagogie hybride » (au sens originel de ce dernier terme) que développent ces instituts. De même que sont explorées les tensions que cette pédagogie peut engendrer sur le terrain de la formation, dans les représentations différentes que les partenaires (syndicalistes et enseignants universitaires) se font des thématiques à valoriser dans les programmes ainsi que des apports qu'ils jugent essentiels non seulement pour mieux savoir mais aussi pour mieux agir. "Savoir pour agir...", ainsi une stagiaire d'une session FO évalue-t-elle l'intérêt de la formation à laquelle elle a participé (cf. p. 156). L'expression est assurément consonante avec les objectifs que les instituts s'assignent et avec les moyens qu'en général ils privilégient. Néanmoins, leur définition et leur mise en application, sont souvent objets d'interrogations sinon de litiges et susceptibles - l'auteur y insiste - d'instrumentalisation par la sphère politique. Si les principes de base du droit à la formation et à l'éducation ouvrières résistent et prévalent dans tous les instituts, il n'en demeure pas moins que les modes d'organisation, de gestion, d'insertion de chacun dans son environnement sont éminemment variables. Ainsi, à partir de l'exemple de l'institut de Grenoble dont elle fournit une étude serrée dans le chapitre 6, l'auteur conclut-elle: «la diversité des formes prises par cette institution universitaire hétéronome pourrait donner lieu à autant de monographies que d'instituts. Elles montreraient que les interprétations du modèle étaient arrimées aux contraintes de l'espace socioéconomique dans lequel ils s'implantaient et aux caractéristiques des personnes qui les mettent en œuvre». Comme le chapitre 7 en donne des illustrations, cette diversité, les choix d'implantation des instituts sur le territoire sont toujours amplement subordonnés à des décisions négociées au plus haut niveau par le ministère du Travail et les différentes centrales syndicales. Les stratégies, les attentes, les visées de ces partenaires ne sont - sans que cela étonne - que très partiellement convergentes, dans la mesure où elles sont sous-tendues par des conceptions hétérogènes des changements et du rôle des mouvements sociaux, des syndicats, de la formation dans l'orientation qu'ils peuvent prendre. 
4 À l'évidence, un tel ouvrage par les faits qu'il rassemble et rend intelligibles, par les questions qu'il traite ou que, volontairement, il laisse ouvertes, par sa disponibilité à la discussion est apte à susciter de nouvelles recherches sur ce "segment» de la formation syndicale.

5 Travail de sociologie il est, indissociablement, un travail de "mémoire historienne » qui n'ignore pas, à côté des documents "objectifs" qu'il réunit, "l'expérience irremplaçable de ceux qui ont vécu les événements ", qui accomplissent leur devoir de mémoire sans que soit négligée l'exigence de vérité au cœur du travail de l'historien. Perspective que J.-P. Vernant défend dans son livre Entre mythe et politique: 2. La traversée des frontières (Paris : Éd. du Seuil, p. 127 sqq). Documents d'archives, compterendu de réunions, témoignages écrits et oraux d'acteurs des instituts contribuent à la réalisation de ce travail de sociologue et d'historienne que Lucie Tanguy propose dans son ouvrage.

\section{AUTEURS}

\section{ALAIN BAUBION-BROYE}

Université Toulouse 2-Le Mirail 